\title{
ATOMIC AND MOLECULAR DATA NEEDED FOR ANALYSIS OF INFRARED SPECTRA FROM ISO AND SIRTF
}

\author{
P.F. BERNATH \\ Depts. of Chemistry and Physics \\ University of Waterloo \\ Waterloo, Ontario, Canada N2L $3 G 1$
}

\begin{abstract}
The satellites ISO and SIRTF are cryogenically-cooled infrared observatories. These telescopes will provide a tremendous increase in sensitivity and will, therefore, require new laboratory data to support their missions. A survey of some of the necessary atomic and moelcular data is presented.
\end{abstract}

ISO and SIRTF are cryogenically-cooled infrared observatories which will operate in earth orbit. They are designed to operate in the 2-200 micron spectral region with moderate resolving power (less than 10,000 for ISO, less than 2,000 for SIRTF) for spectroscopy, photometry and imaging. Because the telescopes are cryogenically-cooled and above the earth's atmosphere, they will provide a spectacular increase in sensitivity unimpeded by telluric absorption.

The acronym ISO stands for Infrared Space Observatory. ISO has a $60 \mathrm{~cm}$ diameter Ritchey-Chrétien telescope cooled to less than $4 \mathrm{~K}$. Launch by the European Space Agency is scheduled for 1993 and ISO has an expected lifetime of 18 months. ISO contains four instruments: a camera (ISOCAM), a long wavelength spectrometer (LWS), short wavelength spectrometer (SWS), and an imaging photopolarimeter (ISOPHOT). The short wavelength spectrometer covers the $2.5-45$ micron $\left(222 \mathrm{~cm}^{-1}-4000 \mathrm{~cm}^{-1}\right)$ range at a resolving power of 2000 or 20,000 (with étalons). The long wavelength spectrometer covers the $45-180$ micron $\left(55-222 \mathrm{~cm}^{-1}\right)$ spectral range with a resolution of 200 or 10,000 (with étalons).

The acronym SIRTF stands for Space Infrared Telescope Facility. SIRTF is the infrared component of NASA Great Observatories program. SIRTF has a $90 \mathrm{~cm}$ primary mirror cooled to $4 \mathrm{~K}$. There are three scientific instruments: an infrared array camera (IRAC), a multiband imaging photometer (MIPS) and an infrared spectrograph (IRS). The infrared spectrograph will operate in the $2.5-200$ micron $\left(50-4000 \mathrm{~cm}^{-1}\right)$ region in low resolution $(\lambda / \Delta \lambda=200)$ and medium resolution $(\lambda / \Delta \lambda=2000)$ modes.

The great sensitivity of ISO and SIRTF will require new laboratory data. For atoms infrared line positions, fine structure intervals and oscillator strengths are largely unknown for neutral elements, let alone their ions. Some of the necessary data can be deduced from the current compilations of atomic energy levels or by ab initio calculation but direct observations are always desirable. One example of the way new data will be needed was the discovery of infrared atomic emission lines in the sun. No laboratory data was available but the lines were eventually assigned to high- $l$ Rydberg transitions of the $\mathrm{Mg}$ atom. 
For molecular species in the mid-infrared new spectra of hot molecules (with temperatures greater than $300 \mathrm{~K}$ ) will be required for "well-known" species such as $\mathrm{H}_{2} \mathrm{O}$, $\mathrm{NH}_{3}, \mathrm{CH}_{4}, \mathrm{CN}, \mathrm{C}_{2}$ and $\mathrm{OH}$. Spectra of new molecules such as hydrocarbon free radicals and ionized polycyclic hydrocarbons are required.

For the far infrared region, laboratory data on molecules are particularly meagre. Pure rotational transitions of metal hydrides and molecular ions such as $\mathrm{HeH}^{+}, \mathrm{OH}^{+}, \mathrm{NH}^{+}$, $\mathrm{CO}^{+}$and $\mathrm{H}_{2} \mathrm{O}^{+}$will be required.

For the study of the spectra of planetary atmospheres species such as $\left(\mathrm{H}_{2}\right)_{2}$ have spectra in the far infrared region. It is possible that other van der Waals molecules are also present in planetary atmospheres. 